\section{ON ELEPHANTIASIS.}

By E. Bascome, Esq., Surgeon, London.

Its seat, patholog!, and apperrance; symptoms; operation on hypertrophied scrotum; predisposing causes; treatment.

Turs disease has been variously described, some writers confounding it with leprosy, others considering it as a primary malady; much confusion has consequently arisen from the misapplication of the term, elephantiasis distinguished for the greatly increased size of the parts involved being entirely and totally distinct from leprosy.

Elephantiasis I take to be rather the sequelæo or degeneration of other disease, its appellation originating, doubtless, in the disfiguration and hideous magnitude resembling the $\lim b$ of an elephant. Although the lower extremities and scrotum are more frequently the seat of this disease, it often attacks the upper extremities, involving sometimes the pectoral muscles-it also attacks the labia pudendi in females. From observation during a long residence in British Guiana, where the disease is rife, I am induced to think the hypertrophy is caused in two ways-viz, from exysipelas, and from a want of tone in the lymphatics, induced by a sort of sub-inflammation in them; that arising from erysipelas, as is generally the case in that malady in the West Indies, being more or less complicated with visceral disease; that from the latter seldom or never so, being more of a local nature, a greater susceptibility to which is observable in those of a lymphatic temperament. When the sequelæe of erysipelas, it is not until after repeated attacks of that disease, and persistence longer of each attack, that enlargement of the part ensues, the inflammatory action at each accession seemingly penetrating deeper, involving the whole of the subcutaneous cellular substance, producing infiltration of that tissue, and, ultimately, extensive partial disorganization, which, degenerating into a variety of shapes, becomes studded with stone-like excrescences or protuberances, intersected, especially about the instep and ankle, with weeping fissures or rhagades, partaking of a sandy or horny appearance. When it commences in the lymphatics, a weariness is felt along their course in the limb, and a benumbed sensation, as though (as it is termed) asleep; a dull, deep-seated, gnawing pain is always present, causing a sickening at the stomach, amounting sometimes to vomiting, and unfitting the patient for general exertion. It is unattended by fever or discoloration, (rosy-blush, as in erysipelas, the pulse is small, feeble, and frequent; tongue pale, or white; and the limb imperceptibly enlarges, assuming a doughy feel, the temperature being lower than natural.

Should the scrotum be fastened on, a puffiness and intolerable itchiness are present, and if the surface be abraded by scratching or otherwise, lymph exudes profusely therefrom, forming crusts, which, adhering firmly, induce an unpleasant prickly sensation when in contact with the dress. The parts by-and-by become thickened into lumps, and run into one confused mass, attaining, in some cases, to an enormous size, with so little sensation as to admit of its being kicked at like a foot-ball; in one case, when amputated, the scrotum weighed ninety-two pounds avoirdupois, filling a pork-barrel!

The testes are usually sound, - -at least, are not necessarily implicated: on one occasion I had to remove one of the testes, it being schirrous from a blow received some years previously to the scrotum becoming enlarged, as the patient himself explained. Hydrocele is, however, a pretty frequent
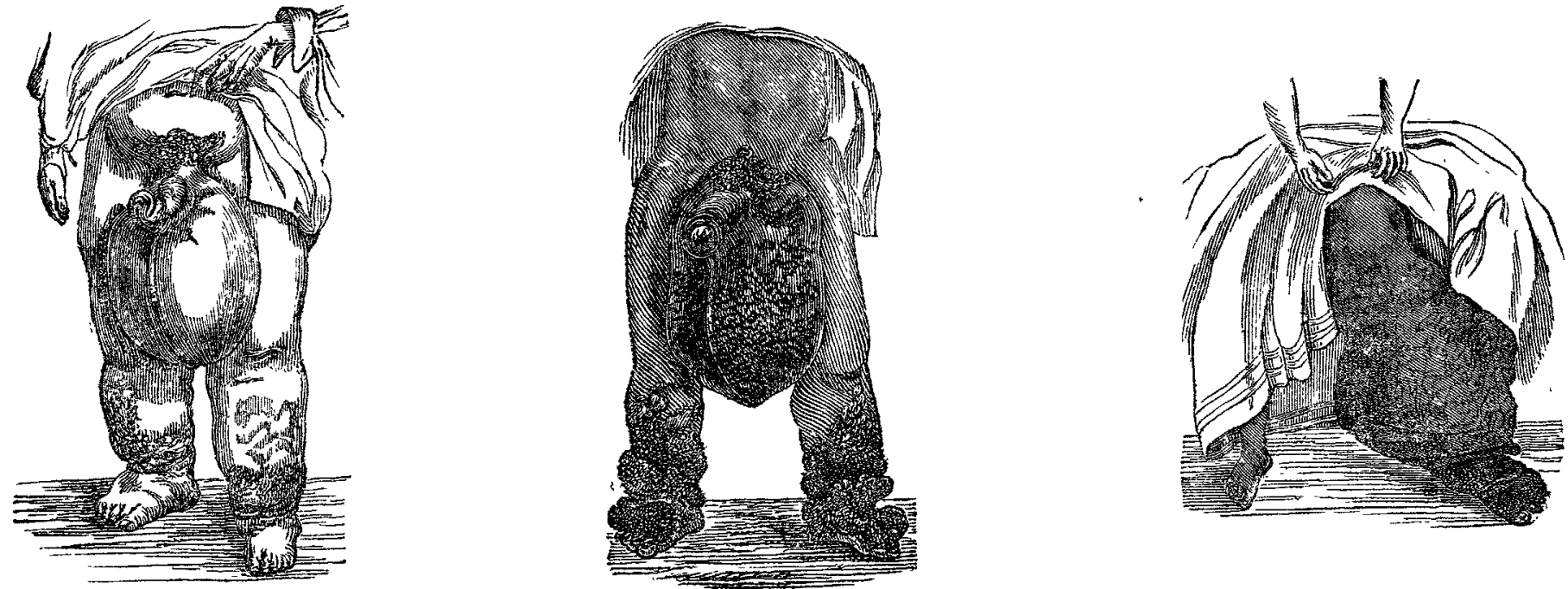

concomitant, but requires no other treatment at the time of amputation than the excision of a sufficient portion of the tunica raginalis.

In operating for the removal of an hypertrophied scrotum, the operator should be guided in the form of his incisions principally by two circumstances-the size and shape of the tumour at its neck, and the position of the navel-like opening of the prepuce, leaving sufficient integuments of the scrotum for the protection of the testes as of the penis for its covering.

In three cases in which I operated, the parts healed by the first intention, and when so healed, I found no difficulty in bringing the integuments saved to the scrotum and penis to a tolerably healthy state by means of frequent superficial scarifications, the application of heated air, and the internal exhibition, three times in the day, of the hydriodate of potash, in ten-grain doses, taken in some carminative vehicle, such as a warm weak infusion of ginger; the parts about the penis and neck of the tumour partaking less of the hypertrophy.

On the dissection of a leg, which $I$ amputated above the knee, the disease seemed to have been seated in the subcutaneous cellular tissue, the muscles, ligaments, \&c., being apparently comparatively sound, the infiltrated or hypertrophied substance at the back of the leg, the calf, measuring six inches and a half in depth or thickness, consisting of a fatty bacon-like substance, with few bleeding vessels. I should state, however, that the bloodvessels (veins) above the knee, where the knife was applied, were unusually large, admitting, at the onset of the operation, of such a rush of blood as to startle one for the moment with the idea that the artery was not properly under command.
On the inspection of a scrotal tumour, in which the substance was very similar to that of the leg, it was interspersed with hydatid-like forms, from which, when cut into, a tenacious transparent fluid escaped.

$I$ have said that $I$ consider peculiarity of habit a predisposing cause; another may be found in the general relaxation and debility of the system from climatic influence.

The exciting cause $I$ attribute to repercussion of cutaneous exhalation from cold air, or cold ablutions of the genitals and lower extremities-in fact, of the entire body, so common in warm climates, in the evening, after the exhausting efforts of the day, the extremities being with the feet uncovered, exposed, during that time, to the direct heat of a burning soil, and surrounded by the heated exhalations therefrom.

With reference to the treatment of this disease, little or nothing can be done when it has arrived at that stage, properly speaking, termed Elephantiasis, disorganization to too great an extent having already taken place.

If the lymphatics be the seat of the malady, rest in the recumbent posture is of the first consideration, but unfortunately seldom or never attended to until it be too late. Cold spirituous evaporating lotions, applied by means of flannel,* will be found grateful to the patient, and afford great relief. Gentle aperients should be administered, purgatives being decidedly injurious. After the subsidence of inflammatory action, a flannel roller, properly applied, so as to support and

* Flannel is recommended as being preferable to either linen or cotton rag; not drying so soon as efther, and for keeping a larger quantity of the lotion like a sponge; at the same time, not interfering with the evaporation nor heating the part. 
give tone to the valvular conformation of the lymphatics, as well as the surcharged cellular tissue, should be had recourse to. At the same time, the internal exhibition of the ioduret of iron, or hydriodate of potass, in some warm carminative, and friction, by means of the flesh-brush, or horsehair glove, with heated air, occasionally medicated with sulphur, will be found excellent adjuvants. When speaking of the bandage, it mast be understood that support, not compression, is wanted, the injudicious application of the roller, or laced stocking, often doing much harm, by causing an extension of the malady to parts seated higher up.

In incipient attacks of the scrotum free superficial incisions, by means of the cupping scarificator, should be persevered in to the removal of the serous deposit; and astringent cooling lotions, such as the chloride of lime, sulphate of zinc, \&c., diligently applied, the internal treatment being that observed when the extremities are affected.

In conclusion, unless those who are so unfortunate as to suffer from this malady be in circumstances to enable them to guard against cold, \&c., by keeping the parts properly protected by clathing, shoes, \&c., very little, or nothing, may be expected from any mode of treatment.

Craven-street, Strand, March, 1846.

ILLUSTRATIONS OF
INFLAMMATION, ULCERATION, \& INDURATION
OERHE TIX UTERI.

Bx J. HENRY BENNET, M.D.,

MEMBER OF THE ROYAL COLLEGE OF PHYSICIANS, LONDON; OBSTETRIC PHYSICIAN TO THE WESTERN GENERAL DISPENSARY, KTC.

DEEP CAOTERIZATION WITII POTASSA FUSA.

Chronic inflammation, ulceration, and induration of the uterine neck in a young married woman, without family, treated by deep cauterization; severe partial prolapsus. Very rare case.

Ar the beginning of May, 1845, I was consulted by Mrs. Ga young married lady, aged twenty-two, residing in London. She was of good constitution, and had always enjoyed perfect health until her marriage, which took place four years previous, at the age of eighteen. She had never conceived. Shortly after marriage, she was attacked with leucorrhoea, and menstruation, which had previously always been easy and free from pain, became difficult. This state of things continued for about a year. She then began to feel a sensation of pelvic weight and bearing-down on walking, and after any exertion. This feeling gradually increased, until one day, after a long walk, she felt the womb fall down quite to the external parts. Being alarmed, and in considerable pain, she returned home, went to bed, and sent for her medical attendant. On examining her digitally, he told her that she was labouring under prolapsus of the uterus. He then returned the prolapsed uterus, kept her in bed for a week, forbidding every kind of exertion. The former bearing-down sensations, however, returned as soon as she got up, and very shortly afterwards, the same accident-the falling of the uterus down to the vulva, again occurred, after fatigue; it was treated as before. From this time forward, the uterus always remained very low, and on any great exertion appeared, she states, at the orifice of the vulva. The leucorrhoal discharge continued to manifest itself, and was occasionally streaked with blood; she experienced also pains in the hypogastric and lumbar regions; menstruation was painful, and her general health became disordered; congress was not painful. After suffering in this manner during a couple of years, without benefiting from medical advice, she was advised to go to Paris, to consult M. Lisfianc.

She accordingly left town in September, 1844, and on her arrival in Paris, placed herself under the above-named celebrated French surgeon. By him she was told that she was suffering from engorgement and ulceration of the neck of the uterus, and placed under his usual treatment. This treatment consists, principally, in rest, occasional cauterization of the vlcerated surface with the nitrate of silver, emollient vaginal injections, and monthly small bleedings from the arm. Finding herself pretty nearly in the same state as at first, after four months of this treatment, she got tired, and applied to M. Ricord. This surgeon, after giving the same diagnosis as M. Lisfranc, also cauterized the ulcerated surface occasionally with a fluid caustic, and prescribed vaginal injections with the nitrate of silver, and complete rest. After three months treatment he pronounced her cured, and she returned to her husband in London, much improved in health.
It was a few weeks after her return that I saw her. She wished to know whether the cure which $M$. Ricord liad announced was real and permanent, as since her return she had experienced many of her former sensations. It appeared that when she left Paris all leucorrhceal discharge had ceased, also the lumbar pains, and that she did not experience the sensation of the uterus being low; she had then been lying on a sofa for months. This sensation, however, returned soon after she got back, and this it was that induced her to consult me.

On examining per vaginam, I found the cervix low down in the vagina, not more than an inch and a half from its ex. ternal orifice; it was slightly retroverted, very voluminous, as large as an egg, perfectly smootl and equal, and extremely resistant to the touch. There was no velvety, mossy sensation. On introducing the middle-sized conical speculum, I found that it exposed only part of the organ: I was obliged to use the bi-valve one, in order to uncover the os and inferior lip, owing to the very large size of the superior one. There was no ulceration, but around the os, especially on the superior lip, in an extent as large as half-a-crown, there was the trace of a former ulceration. This region, instead of being of a rosy white, as it would have been had the tissues underneath been healthy, was of a livid violet hue. The surrounding part of the cervix was deeply congested, and of a vivid red colour.

The cervix uteri was evidently hypertrophied to an extreme degree, and still the seat of chronic inflammation. 'The Paris surgeons had cured the ulceration, but they had left behind the inflammatory induration and hypertrophy which compli. cated it. 'They had therefore merely overcome one of the elements of the disease, and it was evident to me that the nlceration would soon reappear when the patient reassumed the duties of her position; that is, if the organ was allowed to remain in the state it then was. The livid surface which the cervix presented might, indeed, be compared to the cicatrix which forms on a chronically inflamed and ulcerated varicose leg after great care and nursing, and which breaks out again as soon as the patient resumes his occupations. I could not, however, make up my mind at once to propose to this lady, who had been just treated, lying on her back on a sofa for more than seven months, and had been pronounced cured by an eminent surgeon only a few weeks before, to again submit to a tedious and harassing treatment. I was also dubious how far my opinion would be complied with. I therefore told her candidly the state of things; that she was only half cured, that the womb was still too large, and that that was the reason it fell; that she might get completely well, but that I scarcely anticipated such an event. At the same time $I$ advised her to use frequently cold water vaginal injections, not to exert herself in any way; and should the leucorrhœal discharge return, or the lumbar pains and prolapsus increase, to consult me again on the subject. I did not advise a pessary, because I felt cer tain that its contact with the cervix would only accelerate the ulcerating of the mucous surface. Besides, according to my view, the course to be followed in such a case is clear. No mere palliative method of treatment ought to be adopted, but the disease, if seriously treated, ought to be attacked by those measures only which are capable of removing it entirely. In my own mind I felt pretty certain, that in delaying the treatment I was only giving the patient a little respite-a little breathing time.

As I had anticipated, on the 7 th of August, two months after I had first seen Mrs. G__, she called upon me, and stated that the discharge had retmmed, although not very abundantly; that she experienced considerable uterine and lumbar pains, and that whenever she walked the nterus came down very low, which made her feel extremely ill. On exa. mining with the speculum, I found that the livid surface of the cervix had ulcerated in its entire extent, as I had anticipated it would, and was secreting pus abundantly. The adjacent mucous surfaces of the cervix and of the superior legion of the vagina were congested, and in addition to the deepseated, regular, and general hardness of the hypertrophied cervix before recognised, there was a superficial puffiness, which had still further increased in size and weight, and accounted for its still further weighing down the uterus. The indication for treatment was evident. The ulceration was a mere epiphenomenon scarcely worthy of notice. The real disease was the chronic general inflammatory induration of the cervix, and it was that which had to be subdued. Had the disease existed in a woman who had had children, I should not have hesitated a moment to have had recourse to deep cauterization with the potassa fusa at once, as I should have concluded that it alone, or cauterization with the actual cautery, was a sufficiently powerful agent to effect a cure. General and obstinate hypertrophy and induration of the 